\title{
Prediction of infectious disease spread based on cellular automata Donghui Wang ${ }^{1, \text { a }}$ \\ ${ }^{1}$ North China Electric Power University, Baoding, 071000, China \\ apersonalintouch@foxmail.com
}

Keywords: Cellular Automata, Moore expansion neighborhood, distance factor.

\begin{abstract}
Nowadays the infectious disease has greatly affected our life. Infected persons and deaths have reached a record high . And it is still in the state of deterioration. Therefore, I try to establish a predictive model of the current virus spread .Aiming at the spread of infectious diseases, Professor Kermack and Professor McKendrick presented the traditional SIR model. But the dynamic simulation of the numerical analysis of the model shows that it needs further improvement. Also the infected process of the patients is not intuitive.Considering all the above factors, I take people in the epidemic area into six states and the terms of isolation rate, infection rate and cure rate are introduced to describe the relations between the six states.By applying the distance factor[1],I broaden the traditional Moore neighborhood.Then the cellular automata model aiming at the infectious disease is established.
\end{abstract}

\section{Introduction}

Cellular automata fit on the spread of infectious disease has a good effect[2]. Based on this, we designed a rectangular grid, where each cell can be regarded as a person, so the whole area of the rectangular area could represent a population-evenly-distributed area. Cellular automata can reflect the states of different people, spread of disease trend and cycle, so in terms of the prediction of infectious diseases, CA model is a sensitive and effective predicting model. When establishing model, there are some explanations of elements as follows.

\section{Mathematical Analysis}

\subsection{Symbol Definition}

P1 --the probability of mild infection to susceptible

$\mathrm{P} 2-$-isolation rate

P3--the probability of severe infection to susceptible

$\mathrm{P} 4$--cure rate

P5--immune factor

D--distance factor

i--reciprocal of the distance between the cellulars

$\Omega$--sum of reciprocal of the distance between the cellulars

\subsection{The Different States of the Population}

According to the spreading characteristics of infectious disease, different people, with different resistance to infectious diseases, take different effects in the process of the spread of infectious disease, so we divide the study population into six states by adopting the modeling idea of Infectious Disease Dynamics:

- Susceptible(S): they are not infectious, but they are easy to be infected.

- Mildly infected(M): they are in the early days of infection, and have infection with certain capabilities. In a cycle, mild infected have different probability $\mathrm{P}_{1}$, based on the distance between susceptible people and mildly infected, to transform them to mild infected.. Meanwhile, due to the unconspicuous symptoms, they have some probability $\mathrm{P}_{2}$ to be found and isolated; otherwise, in next cycle, they will become severely infected. 
- Severely infected(I): they are in an advanced state of disease and have high infectivity. In a cycle, severely infected have different probability $\mathrm{P}_{2}$, based on the distance of susceptible people, to transform them to severely infected. Meanwhile, due to the obvious symptoms, they will be isolated

- Isolator(Q): they are transformed from those who are mildly infected or severely infected in last cycle. They are isolated.So they cannot contact with outsiders and they are not infectious. Based on different medical measures, they could recover with the probability $\mathrm{P}_{4}$; otherwise, the deterioration of the disease leads them to death.

- Recovered(R): they have long-term immunity, and cannot be infected in a short time.

- Death(D): isolators will die with the deterioration of the disease. They exit the process of the spread of infectious diseases

The relation of the six states is as follows:

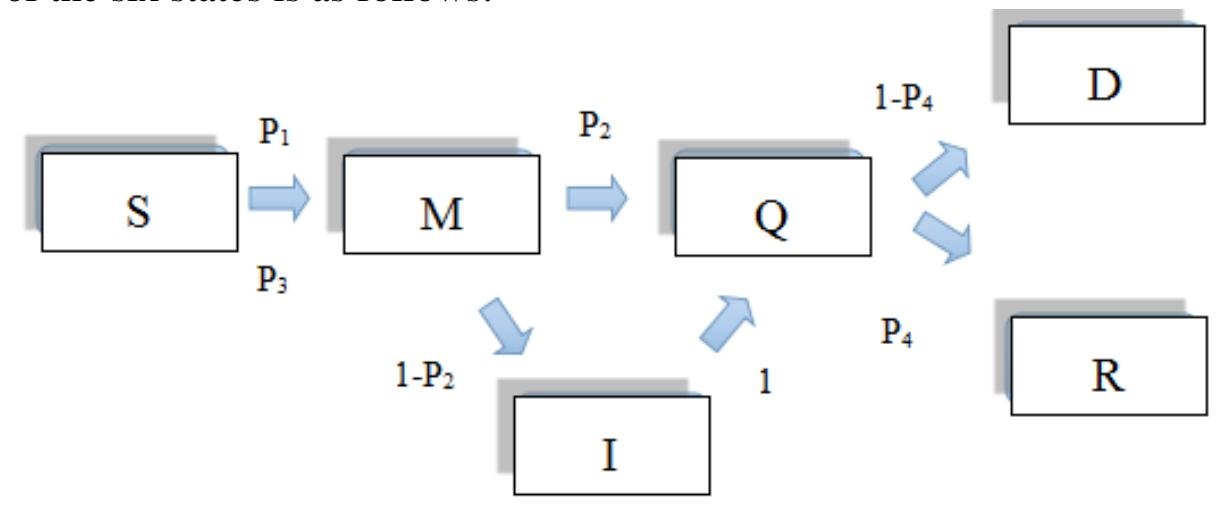

Figure 1: The relationship between the six states

\subsection{Infection Parameter}

In real life, there is a great difference between individuals[3], and susceptible to diseases have different resistance $R_{i, j}$. Mildly infected and severely infected have different capability $f_{i, j}$ of infection. The spread of infectious disease is related to distance: the greater the distance between the contacts, the lower the probability of being infected by each other. In order to introduce effective contagious probability $\mathrm{P}_{1}, \mathrm{P}_{3}$ (the formula of the following is $\mathrm{P}_{\mathrm{i}, \mathrm{j}}$ ), we introduce distance factor:

$D_{C_{i, j} C_{i^{\prime}, j^{\prime}}}=\frac{i_{C_{i, j} C_{i^{\prime}, j^{\prime}}}}{\Omega_{C_{i, j}}}$

$\mathrm{C}_{\mathrm{i}, \mathrm{j}}$ : the central cell

$\mathrm{C}_{\mathrm{i}, \mathrm{j}}$ : a neighbor in its Moore neighborhood

Where two variables above equal :

$$
\begin{aligned}
& i_{C_{i, j} C_{i^{\prime}, j^{\prime}}}=\frac{1}{\sqrt{\left(i-i^{\prime}\right)^{2}+\left(j-j^{\prime}\right)^{2}}} \\
& \Omega_{C_{i, j}}=\sum_{C_{i, j \neq C_{i^{\prime}, j^{\prime}}}}^{N_{C_{i, j}}} \frac{1}{\sqrt{\left(i-i^{\prime}\right)^{2}+\left(j-j^{\prime}\right)^{2}}}
\end{aligned}
$$

$N_{C_{i, j}}$ : all neighbor collection in the Moore neighborhood of the central cell $\mathrm{C}_{\mathrm{i}, \mathrm{j}}$

Combining these factors, the effective contagious probability $\mathrm{P}_{\mathrm{i}, \mathrm{j}}$ of the central cell $\mathrm{C}_{\mathrm{i}, \mathrm{j}}$ is:

$$
P_{i, j}=\sum_{C_{i, j} \neq C_{i^{\prime}, j^{\prime}}}^{N_{i, j}} D_{C_{i, j} C_{i^{\prime}, j^{\prime}}} \times \sqrt{f_{i, j} \times\left(1-R_{i, j}\right)}
$$

the higher the value of $\mathrm{P}_{\mathrm{i}, \mathrm{j}}$, the more likely to get sick. 


\section{Comprehensive Analysis}

$\{0,1,2,3,4,5\}$ respectively represent a cell six states at time $t$, and assign them in different colors, so we can derive spread trend of infectious diseases through image analysis and statistics of the corresponding figures. The number corresponding to each state and colors are as follows:

Table 1 Different symbols

\begin{tabular}{ccc}
\hline State & Number & Color \\
\hline Susceptible & 0 & \\
Mildly infected & 1 & \\
Severely infected & 2 & \\
Isolator & 3 & \\
Recovered & 4 & \\
Death & 5 & \\
\hline
\end{tabular}

This paper use the expansion Moore neighborhood, so sick of CA model Moore neighborhood have different infectious to such as family members, neighbors, and people outside the area adjacent Moore. Through the introduction of the distance factor 4.3 shows, this probability will decreases with increasing distance :

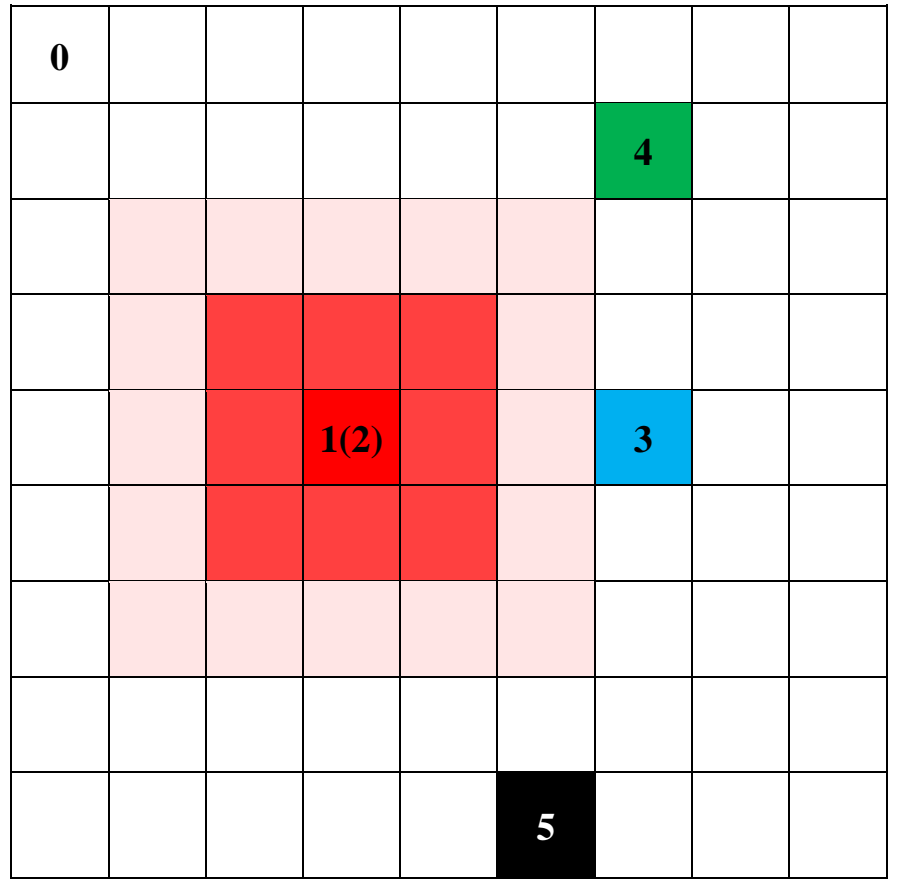

Figure 2: The expansion Moore neighborhood

\section{Conclusion}

Through all the above analysis ,I successfully establish the cellular automata model aiming at the infectious disease spread.By taking people in the epidemic area into six states,we can clearly catch the spread between different people.Its result is closely to the realistic situation.Also,this model is the basic of the research to cure infectious disease.We can take further analysis to develop a reasonable vaccination program. 


\section{References}

[1]Chao Guan, Wenyan Yuan, Yun Peng. A Cellular Automaton Model with Extended Neighborhood for Epidemic Propagation. Fourth International Joint Conference on Computational Sciences and Optimization, 2011, ISBN: 978-0-7695-4335-2,p. 623-627.

[2]Jiatai Gang, Qinwu Bin, Shujuan Li, Xinxin Tan. Infectious diseases based on cellular automata model with isolated intervention.Journal Of Dalian University 2014(3),p.1065-1068

[3]G Ch Sirakoulis, I Karafyllidis, A Thanailakis. A cellular automaton for the effects of populaton movement and vaccination on epidemic propagation. Ecological Modeling, Vol.133(2000)NO. 3, p.133: 209-233. 